\title{
Carbohydrates surge through clinical trials
}

FT. COLLINS, Colo.-Among the dozen or so biotech companies focusing on complex carbohydrates, perhaps the furthest along are Alpha-Beta Technology (Worcester, MA), Cytel (San Diego, CA), Genetics Institute (Cambridge, MA), Gliatech (Cleveland, $\mathrm{OH}$ ), Glycomed (Alameda, CA), Oxford GlycoSystems (Alameda, CA), and Telios Pharmaceuticals (San Diego, CA). A number of pharmaceutical firms-including Eli Lilly (Indianapolis, IN), Merck (Rahway, NJ), and SmithKline Beecham (King of Prussia, PA)have also mounted major carbohydrate research efforts.

Carbohydrate-based drugs already exist. The anticoagulant heparin is a complex carbohydrate. So, too, are fiber supplements such as Meta-mucil and Fiber-Con. What is new about the carbohydrate-based drugs now being developed in biotech labs is that they are tailor made to either augment or interfere with the specific cellsurface receptors that initiate cell adhesion.

Cell adhesion encompasses a broad range of molecular interactions, most of which involve carbohydrates, that allow cells to recognize and stick to one another. Such processes are important in immune responses, inflammation, cancer metastasis, blood clotting, wound healing, scarring, and other biochemical events. What has become clear over the past decade is that the surface of each cell contains any number of adhesion molecules, each of which is involved in a specific cell-cell or cell-molecule recognition process. It is this specificity that has carbohydrate researchers so excited about the therapeutic potential of these compounds.

For example, the tetrasaccharide known as sialyl Lewis ${ }^{x}\left(\operatorname{SLe}^{x}\right)$ is found on the surface of neutrophils, the white blood cells involved in producing inflammation. It binds to a specific adhesion receptor on blood vessels that enables neutrophils to migrate from the blood stream into tissues. Cytel has begun two phase I clinical trials with a $S e^{x}$ derivative called CY 503 , one for acute lung inflammation and the other for reperfusion injury. Glycomed, Genetics Institute, and Oxford GlycoSystems are also developing SLe ${ }^{x}$ derivatives for such applications as cancer and inflammation.

Neutrophils also recognize a carbohydrate, known as beta-glucan, on the surface of fungus and yeast, which is a key to their ability to trigger an immune response against these infectious organisms. By modifying the structure of beta-glucan, using genetically engineered yeast cells, AlphaBeta Technology has developed a compound, called betafectin, that acts as an antibiotic by binding to both neutrophils and monocytes, thereby stimulating these cells to bind to yeast and fungus and kill them, without causing an increase in cytokine production. Cytokines are immune-stimulating substances that can triggersuch serious side effects as inflammation and tissue damage during an immune response.

To date, Alpha Beta has completed two phase II trials of betafectin. The first trial showed that prophylatic administration of the compound reduces by 47 percent the incidence of fungal and yeast infections following abdominal surgery. The second trial demonstrated that betafectin reduces fungal and yeast infections in patients with severe burns by 40 to 50 percent. Alpha-Beta is now consulting with the Food and Drug Administration (FDA, Bethesda, MD) about the design of its phase III trials of betafectin, which it hopes to begin before the end of the year.

Another natural carbohydrate, a proteoglycan known as decorin, inhibits transforming growth factorbeta (TGF-beta), a protein that causes cells to overproduce complex carbohydrates and, thus, adhere to immune-system cells. TGF-beta plays a role in wound healing, but the process it induces often goes too far, resulting in scar-tissue formation. In some instances, TGF-betainduced scar formation occurs as a byproduct of various disease processes, and, in fact, such scar formation is the leading form of kidney failure and liver cirrhosis. Telios Pharmaceuticals is testing a modified form of decorin to block TGFbeta's scar-forming activity without interfering with the other functions it plays in tissue repair and immune responsiveness. Last year Telios reported that the compound stopped scarring in an animal modcl of kidney disease. Telios is now completing toxicology studies on the compound and expects to submit an investigational-new-drug application for the compound to the FDA early next year.

One broad class of molecules that appears to hold cells together are the family of carbohydrates known as glycosaminoglycans (GAGs). These large polymers of glucose extend from the surfaces of cells into the waterfilled space between cells, where they mesh with GAG chains from neighboring cells. Researchers at Gliatech have found that GAGs and certain derivatives, perhaps by forming a physical barrier, can not only hold cells together but can exclude other cells from an area. The company is capitalizing on this property to prevent scar-forming cells, called fibroblasts, from entering surgical wounds during the two-week period critical for scar formation.

Animal studies have shown that a chemically modified GAG, when applied as a gel to a surgical incision, stopped fibroblasts from entering the area surrounding an incision without impeding normal wound healing. The adhesion-controlling compound, known as Adcon, is not absorbed into cells and is degraded by naturally existing enzymes and, thus, has been classified by the FDA as a resorbable device. Illustrating the speed at which the carbohydrate field is moving, it took a mere 15 months for Adcon to progress from the first observations in animals to the start of clinical trials. Currently, Adcon is in the final months of pivotal clinical trials for preventing scar formation following surgery to remove damaged spinal disks. Animal studics have begun to demonstrate efficacy in abdominal, pelvic, and cardiac surgeries, as well as tendon-implant and organ-implant surgeries.

-Joseph Alper
Biotech firms

are tailoring

carbohydrates

to either

augment or

interfere with

the specific

receptors that

initiate cell

adhesion. 